\title{
SRAFFA, WITTGENSTEIN \\ Y GRAMSCI
}

Amartya Sen*

$\mathrm{E}$ n febrero de 2003, cuando la Accademia Nazionale dei Lincei hizo en Roma una gran conferencia sobre el vigésimo aniversario de la muerte de Piero Sraffa ${ }^{1}$, honró la memoria de un gran intelectual que publicó muy poco pero influyó notablemente en la economía, la filosofía y las ciencias sociales contemporáneas. Su impacto intelectual comprende nuevas exploraciones en teoría económica, incluida una revaluación de la historia de la economía política (empezando con la obra de David Ricardo) ${ }^{2}$. También tuvo suma influencia en una de las principales reorientaciones de la filosofía contemporánea, el paso trascendental de Ludwig Wittgenstein de su posición inicial en el Tractatus lógicus-philosophicus (1921) a la de Investigaciones filosóficas $(1951)^{3}$.

A menudo se separa al "Sraffa economista" de sus demás facetas. Debido en parte a que profesionalmente Sraffa era economista, y en

* Doctor en Economía, profesor del Departamento de Economía, Universidad de Harvard, Cambridge, Mass., Estados Unidos, [asen@fas.harvard.edu]. Tomado de Journal of Economic Literature 41, 4, 2003, pp. 1240-1255. Traducción de Alberto Supelano. Se publica con las autorizaciones correspondientes. Fecha de recepción: 10 de febrero de 2016, fecha de aceptación: 12 de mayo de 2016. Sugerencia de citación: Sen, A. "Sraffa, Wittgenstein y Gramsci”, Revista de Economía Institucional 18, 34, 2016, pp. 257-280. DOI: http://dx.doi.org/10.18601/01245996.v18n34.14

1 "Piero Sraffa: Convegno internazionale", 11-12 de febrero de 2003. Este artículo se basa en un ensayo más largo que presenté allí (Sen, 2004). Por sus útiles discusiones durante largos años, tengo una gran deuda con K. Arrow, K. Basu, C. Bliss, N. Denyer, M. Dobb, P. Garegnani, F. Hahn, G. Harcourt, J. Hicks, H. Kurz, B. McGuinness, J. Mirrlees, R. Nozick, L. Pasinetti, S. Payne, H. Putnam, J. Robinson, E. Rothschild, R. Solow, L. Spaventa y S. Zamagni, y con el mismo Piero Sraffa. Agradezco también al editor y a los árbitros del JEL por sus útiles sugerencias.

${ }^{2}$ Ver Garegnani (1960; 1998), Roncaglia (1978; 1999), Pasinetti (1979; 1988), Kaldor (1984; 1985); Eatwell y Panico (1987); Samuelson (1987; 2000a; 2000b); Sylos Labini (1990) y Schefold (1996).

${ }_{3}^{3}$ Para los temas relacionados con esta transición, ver Wittgenstein $(1953 ; 1958)$. 
parte a que sus contribuciones económicas parecen, al menos superficialmente, apartarse de sus ideas filosóficas. Aunque solo publicó algunos artículos y un libro, además de editar las obras de Ricardo, Sraffa es muy citado en economía ${ }^{4}$. Sus aportes económicos, en particular su único libro, Producción de mercancías por medio de mercancías: preludio a una crítica de la teoría económica (1960), han suscitado importantes controversias. Sus trabajos iniciaron una corriente de pensamiento en teoría económica, aunque otros economistas argumentan que en sus escritos no hay nada sustancial, y aun otros (en especial Paul Samuelson) sostienen que Sraffa es en parte profundo y en parte equivocado ${ }^{5}$.

Es fuerte la tentación de examinar al "Sraffa economista" por separado. Pero hay algo que ganar si sus aportes se consideran en conjunto. No menos importante para la historia del pensamiento filosófico puede ser reexaminar la interacción de Sraffa con Wittgenstein -en quien influyó notablemente- a la luz de su relación con Antonio Gramsci, el teórico marxista, quien tuvo gran influencia en Sraffa. De hecho, esa relación dual también da la oportunidad de explorar una posible “conexión Gramsci” en la transformación del "primer Wittgenstein” en el "segundo Wittgenstein".

\section{WITTGENSTEIN Y SRAFFA}

Ludwig Wittgenstein regresó al Trinity College, en enero de 1929, después de haber salido de Cambridge en 1913, donde fue estudiante de Bertrand Russell. El regreso de Wittgenstein fue todo un acontecimiento, dada su reputación como filósofo genial. John Maynard Keynes escribió a su mujer, Lydia Lopokova: “Bien, Dios llegó. Lo conocí en el tren de las 5:15".

Piero Sraffa, quien no conocía a Wittgenstein, se trasladó a Cambridge desde Italia casi un año antes del regreso de Wittgenstein. Sraffa solo tenía 29 años (nació en Turín el 5 de agosto de 1898) pero ya era muy conocido en Gran Bretaña y en Italia como un economista muy original. Obtuvo un grado de investigación en la Universidad de Turín a finales de 1920, con una tesis sobre economía monetaria, pero fue un artículo sobre los fundamentos de la teoría de los precios que publicó en 1925 en Annali di Economia (con sede en Milán) el que lo convirtió en una celebridad en Italia y Gran Bretaña. En ese ensayo

${ }^{4}$ Ver, p. ej., Eatwell et al. (1987). Los libros en inglés sobre la vida y los aportes de Sraffa incluyen, entre otros, Steedman (1977; 1988), Roncaglia (1978), Potier (1987), Schefold (1989), Bharadwaj y Schefold (1990), Cozzi y Marchionatti (2000), y Kurz (2000).

${ }^{5}$ Ver Samuelson (1987; 2000a; 2000b), ver también Frank Han (1982). 
demostró que los fundamentos de la teoría desarrollada por Alfred Marshall (el líder de la entonces dominante escuela de Cambridge) eran irremediablemente defectuosos. Una ampliación significativa de ese ensayo apareció en inglés el año siguiente en Economic Journal (Sraffa, 1926) y fue sumamente influyente.

Sraffa también tenía profundos intereses y compromisos políticos, participaba en el Grupo de Estudiantes Socialistas y se unió al equipo editorial de L'Ordine Nuovo, una publicación de izquierda fundada y editada por Antonio Gramsci en 1919 (que sería prohibida por el gobierno fascista). Cuando Sraffa se mudó a Gran Bretaña en 1927, era una figura importante entre los intelectuales italianos de izquierda, y estaba cerca -aunque no era miembro- del Partido Comunista Italiano, fundado en 1921 y dirigido por Gramsci. Aunque obtuvo el cargo de catedrático en la Universidad de Perugia en 1923, y de profesor en Cagliari en 1926, consideró trasladarse a Gran Bretaña, cuando la persecución fascista se endureció en Italia.

Ya en 1922, el padre de Piero, Angelo Sraffa, entonces rector de la Universidad Bocconi, había recibido dos telegramas de Mussolini exigiendo que Piero se retractara de una descripción crítica de las políticas financieras italianas que había publicado en el Manchester Guardian (por invitación de John Maynard Keynes). "Difundía la desconfianza" y era "un acto de verdadero y real sabotaje", se quejó Mussolini. Angelo Sraffa, un académico valiente y decidido, replicó que el artículo solo mencionaba "hechos conocidos" y que no había nada en particular de qué retractarse. Piero Sraffa tuvo otros altercados con el gobierno italiano en los años siguientes, y acogió con entusiasmo una invitación que le transmitió John Maynard Keynes en una carta de enero de 1927 para ocupar una cátedra en Cambridge. Se trasladó a esta ciudad en septiembre de ese año. Cuando Wittgenstein regresó allí en enero de 1929, Sraffa ya tenía allí una reputación legendaria como uno de los intelectuales más brillantes.

Sraffa influyó en el pensamiento de Wittgenstein mediante una serie de conversaciones regulares ${ }^{6}$. ¿Qué forma tomó esa influencia? Provocó un cambio en el enfoque filosófico de Wittgenstein en los años siguientes, un cambio en el que esas conversaciones tuvieron un papel esencial. En sus primeros trabajos (en particular en el Tractatus logicus-philosophicus), Wittgenstein adoptó un enfoque que a veces se llama "teoría pictórica del significado", en el que una frase representa un estado de cosas porque es una especie de imagen que refleja la estructura del estado de cosas que describe. A riesgo de simplificar en

${ }^{6}$ Ver McGuinness (1982), Ray (1991), Albani (1998) y Davis (2002), entre otros. 
exceso, se puede decir que se insiste en que una proposición y lo que describe deben tener la misma forma lógica. Sraffa consideró que esa posición filosófica era totalmente errónea, y discutió con Wittgenstein la necesidad de que la replanteara.

Según una anécdota famosa, Sraffa respondió a la pretensión de Wittgenstein tocándose el mentón con la punta de los dedos, el gesto napolitano para mostrar escepticismo, y preguntó, “¿cuál es la forma lógica de esto?”. Sraffa (a quien después tuve el privilegio de conocer bien -primero como estudiante y después como colega- en Trinity College) insistía en que esta anécdota, si no del todo apócrifa ("no puedo recordar esa ocasión específica”), era un relato con moraleja más que un hecho real ("Discutí tanto con Wittgenstein que mis dedos poco tenían que hablar"). Pero la historia ilustra el escepticismo de Sraffa hacia la filosofía expuesta en el Tractatus, y en particular que las convenciones sociales pueden contribuir a dar significado a nuestras expresiones y gestos.

Las conversaciones con Sraffa fueron trascendentales para Wittgenstein, quien después diría a Henrik von Wright, el distinguido filósofo finlandés, que esas conversaciones le hicieron sentirse "como un árbol al que se han cortado todas las ramas”. Se suele dividir su obra entre el "primer Wittgenstein" y el "segundo Wittgenstein", y el año 1929 marca la línea divisoria entre esas dos etapas. Sraffa no fue el único crítico a quien Wittgenstein reconoció. Otro fue Frank Ramsey, el joven prodigio matemático de Cambridge. Wittgenstein agradeció a Ramsey (1953, x), pero señaló que tenía una deuda "aun mayor" con la crítica que "un profesor de esta universidad, P. Sraffa, ejerció incesantemente sobre mi pensamiento durante muchos años", y añadió: "A este estímulo le debo las ideas más importantes de este libro".

Wittgenstein le dijo a su amigo Rush Rhees (otro filósofo de Cambridge) que lo más importante que Sraffa le enseñó fue la "manera antropológica” de ver los problemas filosóficos. En su penetrante análisis de la influencia de Sraffa y Freud, Brian McGuinness analiza el impacto de "la manera etnológica o antropológica de ver las cosas proveniente del economista Sraffa" (1982, 36-39). Mientras que el Tractatus considera el lenguaje en forma aislada de las circunstancias sociales en las que se usa, las Investigaciones fllosóficas subrayan las convenciones y reglas que dan significado particular a las expresiones. Es fácil ver la conexión de este punto de vista con lo que se llegó a conocer como "filosofía del lenguaje ordinario".

El escepticismo que transmite el gesto napolitano de tocarse el mentón con la punta de los dedos (así lo haga un niño toscano de Pisa 
nacido en Turín) solo se puede interpretar según las reglas y convenciones establecidas - la "corriente de la vida"- en el mundo napolitano. Wittgenstein usó la expresión “juego de lenguaje” para ilustrar cómo se aprende el uso del lenguaje y el significado de las palabras y los gestos (aunque, en últimas, en un lenguaje real hay mucho más de lo que se puede considerar como juegos de lenguaje).

También podemos imaginar que todo el proceso de uso de palabras [...] es uno de esos juegos por medio de los cuales los niños aprenden su lengua materna. Llamaré a estos juegos “juegos de lenguaje” y a veces hablaré de un lenguaje primitivo como un juego de lenguaje (1953,5).

\section{RESERVA Y RUPTURA}

¿Estaba Sraffa emocionado por el impacto de sus ideas sobre quien fue, sin duda, el principal filósofo de nuestra época ("el Dios" a quien Keynes conoció en el tren de las 5:15)? Además, ¿cómo llegó Sraffa a esas ideas trascendentales? Eso le pregunté más de una vez en nuestros paseos regulares de la tarde entre 1958 y 1963. Sus respuestas eran desconcertantes. No, no estaba emocionado, porque su argumento era "bastante obvio". No, no sabía cómo llegó a esos argumentos, puesto que -de nuevo- el punto era "bastante obvio".

A Sraffa le agradaba Wittgenstein y le tenía mucha admiración ${ }^{7}$. Pero era claro que no estaba convencido de la fecundidad de conversar continuamente con ese filósofo genial. Cuando llegué a estudiar al Trinity a comienzos de los años cincuenta, poco después de la muerte de Wittgenstein, advertí que había algún distanciamiento entre ellos. En respuesta a mis preguntas, Sraffa era reacio a hablar de lo que ocurrió realmente. "Tuve que dejar nuestras conversaciones regulares, me aburrían”, fue lo más cercano a una explicación que logré obtener. Sin embargo, Ray Monk describió los hechos con mayor detalle en su biografía de Wittgenstein:

En mayo de 1946 Piero Sraffa decidió que ya no deseaba tener conversaciones con Wittgenstein, diciendo que ya no podía dedicar su tiempo ni su atención a los asuntos que Wittgenstein deseaba discutir. Eso fue un gran golpe para Wittgenstein. Le rogó a Sraffa que mantuvieran sus conversaciones semanales, aunque eso significara abandonar los temas filosóficos. "Hablaremos de cualquier cosa", le dijo. "Sí”, replicó Sraffa, "pero a su manera" $(1991,487)$.

\footnotetext{
${ }^{7}$ Wittgenstein no solo admiraba a Sraffa, también le confió la custodia de algunos de sus escritos filosóficos. Sraffa le escribió a von Wright el 27 de agosto de 1958 (copia del manuscrito de la carta en la Wren Library del Trinity College): "Al comparar mi copia del libro azul con la edición publicada recientemente [Wittgenstein, 1958] veo que tiene algunas pequeñas correcciones en letra de Wittgenstein que no se tuvieron en cuenta en la versión impresa. Supongo que hizo estas correcciones cuando me dio el libro poco después de la muerte de Skinner [en 1941], a quien perteneció originalmente”.
} 
Hay muchos aspectos enigmáticos en la relación de Sraffa y Wittgenstein. ¿Por qué Sraffa, que amaba el diálogo y la discusión, se volvió tan reacio a hablar con una de las mentes más brillantes del siglo XX? Incluso al comienzo, ¿por qué las conversaciones, que eran tan importantes para Wittgenstein que le hicieron sentirse "como un árbol al que le han cortado todas las ramas", le parecían "bastante obvias" a este economista de Toscana? Dudo que alguna vez estemos seguros de saber la respuesta a esas preguntas. En cuanto a la ruptura posterior, a Sraffa quizá le molestaban los modales dominantes de Wittgenstein (caricaturizados en un poema de un estudiante, Julian Bell, hijo de Clive Bell: “¿quién, en cualquier materia ha visto alguna vez/que Ludwig se abstenga de sentar cátedra? En toda reunión nos acalla a gritos/e interrumpe nuestra frase tartamudeando la suya").

A Sraffa quizá también le exasperaba la ingenuidad política de Wittgenstein. Tuvo que evitar que Wittgenstein -con sus ancestros judíos y su franqueza constitutiva- fuera a Viena en 1938, justo cuando Hitler marchaba triunfante por la ciudad. Además, aunque ambos tenían convicciones políticas de izquierda, Sraffa (realista político avezado) veía poco mérito en las excentricidades de las creencias sociales de Wittgenstein, que combinaban un anhelo romántico por la ardua vida del trabajador manual con la esperanza de que la revolución comunista llevara a rechazar la adoración de la ciencia, a la que Wittgenstein consideraba una influencia corrupta en la vida contemporánea.

Subsiste, sin embargo, la pregunta de por qué Sraffa era tan reservado sobre la profundidad y novedad de sus conversaciones con Wittgenstein aun al comienzo (en 1929 y poco después), y por qué las ideas que tanto influyeron en Wittgenstein a él la parecían muy sencillas. Sraffa no publicó nada sobre este tema, pero hay muchas pruebas de que lo que a Wittgenstein le parecía una nueva sabiduría era un tema común de discusión en el círculo intelectual al que pertenecían Sraffa y Gramsci. Trato este tema a continuación.

\section{LA CONEXIÓN GRAMSCI}

Antonio Gramsci era menos reacio a poner por escrito sus ideas filosóficas. Cuando Keynes le escribió a Sraffa en enero de 1927 para comunicarle el ofrecimiento de una cátedra en la Universidad de Cambridge, Gramsci acababa de ser arrestado (el 8 de noviembre de 1926, para ser exacto). Después de algunas experiencias desoladoras en prisión, sobre todo en Milán, fue sometido a juicio en Roma, junto con otros presos políticos, en el verano de 1928. Gramsci fue sentenciado a veinte años de cárcel ("debemos impedir que este cerebro funcione 
durante veinte años", dijo el fiscal en un comunicado que logró cierta fama), y fue enviado a prisión en Turi, a unas veinte millas de Bari. Desde febrero de 1929 se dedicó a escribir ensayos y notas que más tarde serían sus famosas Cartas de la cárcel (1971, 1975).

Esas notas nos dan gran comprensión de aquello que interesaba a Gramsci y su círculo. Sraffa tenía mucho interés en que Gramsci escribiera sus reflexiones, y para ayudarle le abrió una cuenta ilimitada en una librería de Milán (Sperling \& Kupfer), que Sraffa pagaría. Como ya se mencionó, Sraffa era parte del equipo editorial de L'Ordine Nuovo. Se unió al equipo en 1921, pero conocía a Gramsci desde antes y escribía para L'Ordine Nuovo desde 1919 (sobre todo traduciendo trabajos del francés, el inglés y el alemán). El trabajo conjunto en esa distinguida publicación los acercó aún más, y tuvieron intensas discusiones durante años ${ }^{8}$. Aunque discrepaban de vez en cuando; por ejemplo, en 1924, cuando Sraffa criticó la línea del partido (el Partido Comunista "comete un tremendo error porque da la impresión de que está saboteando una alianza de movimientos de oposición”), no hay duda del carácter sumamente productivo de su interacción.

Puesto que las Cartas de la cárcel eran, en muchos sentidos, una continuación de la larga búsqueda intelectual de Gramsci y reflejaban el tipo de ideas que interesaba a su círculo de amigos, es útil ver cómo se relacionan sus notas con el tema de las conversaciones entre Sraffa y Wittgenstein, incluido el papel de las reglas y convenciones y el alcance de lo que luego se conoció como "filosofía del lenguaje ordinario". En un ensayo sobre "el estudio de la filosofía" Gramsci examina "algunos puntos de referencia preliminares", que incluyen la audaz afirmación de que "es preciso destruir el difundido prejuicio de que la filosofía es algo sumamente difícil por ser la actividad intelectual propia de una categoría particular de especialistas o de filósofos profesionales y sistemáticos”. Gramsci argumentó, en cambio, que "primero se debe mostrar que todos los hombres son filósofos, definiendo los límites y características de la 'filosofía espontánea' propia de todo el mundo".

¿Qué tipo de objeto es esta "filosofía espontánea"? El primer elemento que Gramsci enumera bajo esta categoría es "el lenguaje mismo, el cual es un conjunto de nociones y conceptos determinados, y no simplemente de palabras gramaticalmente vacías de contenido”. E1

\footnotetext{
${ }^{8}$ Sobre la amistad de Gramsci y Sraffa, ver Naldi (2000). Su interacción intelectual cubría gran variedad de temas. John Davis $(1993 ; 2002)$ investigó y esclareció el impacto de las ideas gramscianas de "hegemonía”, "cesarismo" y "praxis" en el pensamiento de Sraffa, y cómo pueden haber influido en Wittgenstein a través de Sraffa. Esas posibles conexiones son más complicadas que las que se consideran en este ensayo, que se ocupa de los asuntos más elementales del significado y la comunicación que están en la base de la filosofía de la corriente principal.
} 
papel de las convenciones y reglas, incluido lo que Wittgenstein llegó a llamar "juegos de lenguaje", y la relevancia de lo que se ha llamado "manera antropológica", que Sraffa defendió ante Wittgenstein, todo ello figura en forma destacada en los Cuadernos de la cárcel:

Por la propia concepción del mundo se pertenece siempre a una agrupación particular, la de todos los elementos sociales que comparten la misma manera de pensar y de obrar. Se es conformista de algún conformismo, se es siempre hombre masa u hombre colectivo (Gramsci, 1975, 324).

Gramsci ilustró el papel de la convención lingüística con varios ejemplos. He aquí uno de ellos:

También se debe recordar el ejemplo contenido en un librito de Bertrand Russell [Los problemas de la filosofía]. Russell dice poco más o menos: "Sin la existencia del hombre en la tierra, no podemos pensar en la existencia de Londres o Edimburgo, pero podemos pensar en la existencia de dos puntos en el espacio, donde hoy están Londres y Edimburgo, uno al norte y uno al sur" [...] Este y Oeste son construcciones arbitrarias y convencionales, es decir, históricas, porque fuera de la historia real cada punto de la tierra es Este y Oeste al mismo tiempo. Esto se puede ver más claramente en el hecho de que estos términos han cristalizado no desde el punto de vista de un hombre melancólico hipotético en general sino desde el punto de vista de las clases cultas europeas que, mediante su hegemonía mundial, los han hecho aceptar en todas partes. Japón es el Lejano Oriente no solo para el europeo sino también para el estadounidense de California y aun para el mismo japonés, quien, a través de la cultura política inglesa, puede llamar Cercano Oriente a Egipto (ibíd., 447).

Qué tan ligadas estaban las ideas de Sraffa y de Gramsci y cómo se influyeron uno a otro son temas de investigación posterior ${ }^{9}$. Pero es razonable argumentar que, de un modo u otro, Sraffa estaba familiarizado con los temas que ocupaban a Gramsci en los años veinte y comienzos de los treinta. No es muy difícil entender por qué el programa del Tractatus de Wittgenstein le pareciera profundamente equivocado, viniendo del círculo intelectual al que perteneció. Tampoco es difícil ver por qué la fecundidad de la "manera antropológica" -tan novedosa y trascendental para Wittgenstein-le pareciera a Sraffa poco menos que evidente.

${ }_{9}$ Debo mencionar, sin embargo, dos temas en los que la correspondencia -o la disonancia- entre sus ideas merece mucha más investigación. El primero se refiere a lo que Saul Kripke (1982) llama "la paradoja de Wittgenstein", citando su afirmación de que "una regla no podía determinar ningún curso de acción, porque todo curso de acción puede hacerse concordar con la regla". Puesto que el "segundo Wittgenstein" se centra en relacionar el significado y la comunicación con el seguimiento de reglas, Kripke dice que esta "paradoja" es "quizá el problema central de las Investigaciones filosóficas" (p. 7). El segundo se refiere a qué tan lejos se debe seguir la "manera antropológica" de ver los problemas filosóficos, en particular si la "costumbre" solo se debe invocar para entender cómo se usa el lenguaje, o ir tan lejos como David Hume cuando argumentó, en un pasaje citado con aprobación por Keynes y Sraffa, que "la guía de la vida" no era la razón "sino la costumbre" (1938, xxx). Para un examen más detallado de estos dos temas, ver Sen (2004). 


\section{VALORACIÓN DEL CAPITAL Y COMUNICACIÓN SOCIAL}

¿Cuál es la incidencia de esas ideas filosóficas (incluida la manera antropológica) discutidas por Sraffa, Gramsci y Wittgenstein en el trabajo de Sraffa sobre teoría económica? En su trabajo inicial, en particular en el aclamado ensayo publicado en italiano en 1925 y en su versión inglesa en 1926 que estableció su reputación, Sraffa demostró que la tendencia teórica, liderada por Alfred Marshall, a interpretar los resultados de mercado como resultado de la competencia pura implica una contradicción interna cuando hay economías de escala en la producción de las firmas. Su análisis suscitó una gran labor de seguimiento del carácter de las economías de escala y del funcionamiento de las formas de mercado no competitivas, empezando por Joan Robinson (1933) y Edward Chamberlin (1933). Esos primeros aportes económicos no parecen girar en torno a los temas filosóficos que después abordarían Wittgenstein, Sraffa o Gramsci.

Pero en Producción de mercancías por medio de mercancías: preludio a una crítica de la teoría económica (1960), los problemas de interpretación son de importancia central. Intentaré ilustrarlo con dos temas que discute ese elegante libro. El primero de ellos se refiere a la agregación del capital y a la idea de capital como factor de producción. La teoría tradicional, a menudo llamada "economía neoclásica", se puede formular a diferentes niveles de agregación. Bienes de capital como la maquinaria y el equipo son, por supuesto, muy diferentes, y cualquier descripción agregada que invoque el "capital" como factor de producción debe implicar una "modelación" agregativa que sea comprensible y discutible en la comunicación social. También existe la muy discutida pretensión de que la productividad incremental del capital (llamada "producto marginal de capital") rige el valor de su tasa de retorno (como la tasa de interés o de ganancia).

Sraffa rebate estas pretensiones. Muestra que, en general, el capital como factor sustituto de producción no se puede definir independientemente de la tasa de interés, y que es difícil que la llamada productividad marginal de capital rija la tasa de interés. De hecho, las técnicas de producción ni siquiera se pueden clasificar como más o menos "intensivas en capital", porque la intensidad de uso del capital, que depende de la tasa de interés, puede revertir repetidamente su clasificación cuando baja la tasa de interés ${ }^{10}$. Este es un importante resultado técnico. Pero, ¿qué diferencia hace?

${ }^{10} \mathrm{Ha}$ habido grandes controversias sobre el significado y el alcance de estos y otros resultados conexos. Ver Robinson (1953-1954), Solow (1955-1956), Garegnani (1960; 1970; 1990), Samuelson (1962; 1966), Pasinetti (1966; 1974), Harcourt (1972), Dobb (1973), Bliss (1975), Steedman (1977; 1988), Burmeister 
Los modelos agregados neoclásicos donde el capital es un factor de producción son irreparablemente defectuosos. Pero no es necesario exponer la teoría neoclásica en forma agregativa. La producción se puede considerar en términos de distintos bienes de capital y dejarlo así. Además, el conocimiento práctico para diseñar políticas que se puede conseguir argumentando en términos agregativos (como el uso de técnicas menos intensivas en capital cuando el trabajo es barato y el costo del capital es alto) no depende de cómo se determinen las tasas de interés ni de un modelo muy específico de valoración del capital ${ }^{11}$. Pero a nivel de teoría pura, la idea de que el interés es la recompensa de la productividad del capital y no el resultado de la explotación del trabajo (o el residuo pasivo que queda de restar al valor del producto el costo de los insumos, incluidos los salarios) puede jugar $-\mathrm{y}$ a menudo ha jugado- un papel importante en el debate político y social sobre el carácter del sistema capitalista. Por ello, no es difícil entender el contexto político y social de la demoledora crítica de Sraffa del capital como factor de producción una vez se comprende el objeto de la crítica y se interpreta conforme a un debate clásico que ha durado varios siglos. Sus conclusiones son una respuesta a una explicación particular-con relevancia normativa- del sistema capitalista, y es allí donde reside la relevancia social de estos resultados técnicos.

Debo confesar que me es difícil estar convencido de que el escepticismo hacia un capitalismo sin trabas debe girar en torno a temas como la utilidad del capital agregado como factor de producción y la productividad que se le atribuye, $y$ no en torno a la vida tensa que el capitalismo puede generar, salvo que sea refrenado y complementado por otras instituciones, a menudo ajenas al mercado. Pero no es difícil entender la amplia visión social y política del análisis de Sraffa y su relevancia argumentativa en el debate sobre la productividad del capital como explicación de la ganancia.

\section{LOS PRECIOS Y DOS SENTIDOS DE LA DETERMINACIÓN}

Paso ahora al segundo ejemplo. Sraffa considera una economía en equilibrio en la medida en que tiene una tasa uniforme de ganancia (o de interés). Muestra que si tomamos una foto de la economía con una descripción de todas las actividades de producción, con insumos y productos observados, y una tasa de interés dada, a partir de esta información solo podemos determinar (en el sentido de encontrar) los (1980), Walsh y Gram (1980), Bharadwaj (1990), Bharadwaj y Schefold (1990), Baranzini y Harcourt (1993), Cozzi y Marchionatti (2000), Kurz (1990) y Cohen y Harcourt (2002).

${ }^{11}$ Como se discute en Sen (1974), reimpreso en Sen (1984). 
precios de las mercancías y la distribución del ingreso entre salarios $\mathrm{e}$ intereses (o ganancias $)^{12}$. Y si consideramos una tasa de interés $-\mathrm{O}$ de ganancia- más y más alta, la tasa de salarios será consistentemente más y más baja. Así podemos obtener una relación salarios-ganancias con pendiente negativa (una descripción casi tranquila de una "guerra de clases" estacionaria), para esa situación de producción dada, y la especificación de la tasa de interés (o de ganancia) o de la tasa de salarios nos permitirá calcular los precios de todas las mercancías.

$\mathrm{El}$ perro que no ladra en este ejercicio es la demanda: vamos directamente de los datos de producción a los precios. En este ejercicio matemático no es necesario invocar las condiciones de demanda de las distintas mercancías, que para este ejercicio analítico son redundantes. En la interpretación de este resultado preciso aparece plenamente el fundamento filosófico del significado y la comunicación. Es muy importante entender qué significa "determinación" en el contexto matemático (o, para decirlo a la "manera antropológica", cómo se entendería en una comunidad matemática), y no se deben confundir los diferentes sentidos en que se puede usar el término. Los críticos de la teoría económica dominante sienten una fuerte tentación a considerar que la "crítica" de Sraffa muestra la redundancia de las condiciones de demanda en la determinación causal de los precios, de modo que socava esa teoría porque da mucha importancia a la demanda y a la utilidad. Robinson (1961) no es la única comentarista que muestra cierta fascinación a seguir ese camino:

cuando se nos da un conjunto de ecuaciones técnicas de producción y una tasa real de salarios uniforme en toda la economía, no hay lugar para ecuaciones de demanda en la determinación de los precios de equilibrio $(1961,57)$.

Pero como todo el cálculo se hace para una foto dada de la producción (con insumos y productos fijos, como en una instantánea de las operaciones productivas de la economía), la pregunta de qué sucedería si cambian las condiciones de demanda-lo que por supuesto puede llevar a cantidades de producción diferentes- no se aborda en este ejercicio ${ }^{13}$.

12 El resultado se mantiene en esta forma simple cuando no hay producción conjunta, cuya presencia haría más compleja la relación pero no inmanejable; ver Schefold (1989).

13 Analicé las distinciones involucradas en Sen (1978). Para un análisis textual de lo que Sraffa hace $-y$ no hace- respecto al papel de la demanda, ver Salvadori (2000). Dado el carácter del ejercicio de Sraffa (con una producción de mercancías dada), es claro por qué afirma -correctamente- que en su análisis no es necesario el supuesto de rendimientos constantes a escala (1960, prólogo). Las características internas de la foto observada pueden, por supuesto, reflejar relaciones de mercado particulares (e incluso del equilibrio subyacente), especialmente para que hayan surgido la tasa de ganancia uniforme y la tasa de salarios universal. Pero Sraffa tiene razón en que no se necesita añadir otro supuesto ( $p$. 
La tendencia a interpretar la determinación matemática como determinación causal puede causar un gran malentendido ${ }^{14}$.

\section{VALOR E IMPORTANCIA DESCRIPTIVA}

Si los resultados de Sraffa nada tienen que decir de la determinación causal, ¿qué les da interés? Esta pregunta se puede responder considerando el carácter de la comunicación social a la que contribuye la obra de Sraffa. Primero, la determinación analítica - no solo la determinación causal- es un tema que mucho interesa a las personas. Su demostración de que una foto instantánea de las condiciones de producción de la economía nos puede decir mucho de los precios posibles no solo es un diagnóstico analítico notable, es también un resultado de gran interés intelectual para quienes quieren pensar en la correspondencia entre cantidades producidas y precios fijados. Gramsci argumentó que todos son filósofos en algún nivel, y quizá se pueda decir que la curiosidad analítica -e incluso matemática- es general, e influye en nuestro pensamiento social. La idea de que es posible averiguar los precios de las mercancías mirando simplemente el "lado de la producción" (insumos y productos) dado, junto con la tasa de interés, es un robusto resultado analítico.

Una segunda razón para interesarse en los resultados de Sraffa es entenderlos en términos de la idea de valor y de su contenido político. En el pensamiento clásico, "el valor" no era solo una manera de llegar a los precios (Smith, Ricardo y Marx discutieron el problema de ir de los valores a los precios), sino también de hacer una descripción de importancia social. Para muchos economistas la idea de "valor" parece ser del todo errónea. Por ejemplo, Robinson invocó la metodología positivista (se la podría calificar de "popperiana de izquierda") para restar toda relevancia real a la idea de valor en general y a su empleo en la economía marxista en particular. En su Filosofía económica, Robinson hizo así su denuncia:

En este plano la totalidad del argumento parece ser metafísica; es un ejemplo típico del modo en que funcionan las ideas metafísicas. Lógicamente es una

ej., rendimientos constantes a escala) a lo que ya incluye la foto observada (sin considerar cambios contrafactuales).

${ }^{14}$ Sraffa analiza una distinción correspondiente en una nota inédita escrita en 1942 (agradezco a Heinz Kurz por llamarme la atención sobre esa nota): "Este escrito [el libro por publicarse] trata un problema sumamente elemental; tan elemental que su solución por lo general se da por sentada. El problema es determinar las condiciones de equilibrio de un sistema de precios y la tasa de ganancias, independientemente del estudio de las fuerzas que pueden llevar a ese estado de equilibrio" (D3/12/15:2, colección Sraffa, Wren Library, Trinity College, cursivas añadidas). 
mera sarta de palabras, pero para Marx era una fuente de iluminación y para los marxistas actuales, una fuente de inspiración $(1964,39)$.

"E1 valor no ayudará”, concluyó Robinson. "No tiene contenido operativo. Es solo una palabra".

Los problemas filosóficos que plantearon Gramsci y Sraffa, y por supuesto Wittgenstein, tienen gran influencia en esta cuestión. Así como la metodología positivista emite afirmaciones carentes de sentido cuando no se ajustan al sentido estricto de "significado" en los términos limitados de verificación o falsación, el Tractatus también veía poco contenido en afirmaciones que no representan o reflejan un estado de cosas en la misma forma lógica. Como dijo Simon Blackburn, esto implica negar "significado factual o cognitivo a oraciones cuya función no se ajusta a su concepción de representación, como las que se refieren a la ética, al significado o al sí mismo" $(1994,401)$. En cambio, el enfoque filosófico del "segundo Wittgenstein", en el que influyó Sraffa, considera el significado en términos mucho más amplios ${ }^{15}$.

La interpretación del valor y su relevancia descriptiva ha sido bien tratada por Maurice Dobb $(1937,1973)$, el economista marxista que fue amigo íntimo y colaborador de Sraffa durante el largo tiempo de edición de las obras completas de Ricardo. Dobb señaló el interés social y político de una descripción significativa de las relaciones económicas entre personas. Incluso nociones como "explotación”, que para algunos (incluida Robinson) parecen tan "metafísicas", se pueden considerar como un intento de reflejar, en lenguaje comunicativo, una preocupación pública común por las asimetrías sociales en las relaciones económicas. Como dijo Dobb:

“explotación” no es algo metafísico ni un simple juicio "ético" (aún menos "puro ruido") como se ha dicho a veces: es una descripción factual de una relación socio-económica, tal como lo es la apropiada caracterización de Marc Bloch del feudalismo como un sistema donde los señores feudales "vivían del trabajo de otros hombres" $(1973,45)$.

Aunque el análisis de Sraffa de las relaciones de producción y de la coherencia entre costos y precios (en una foto de la economía) difiere de la descripción basada en el trabajo al estilo marxista, es también un

${ }^{15}$ En epistemología hay un problema relacionado sobre el grado de precisión que se necesitaría para que un posible enunciado científico se considere apropiado. El análisis de Sraffa también guarda relación con este problema, de acuerdo con la afirmación de Aristóteles, en Ética a Nicómaco, de que debemos "buscar precisión en cada tipo de cosas hasta donde la naturaleza del tema la admita". Sobre este problema, ver Sen (1982, ensayo 20: "La descripción como elección") y Coates (1996), junto con las referencias citadas allí. Sin embargo, aquí no profundizaré en ese tema. 
intento de expresar las relaciones sociales centrándose en la producción, y no en la utilidad y las condiciones mentales. Podemos debatir cuán profunda es esta perspectiva, pero es importante ver que el objeto del análisis de Sraffa es una descripción esclarecedora de los precios y la distribución del ingreso, que solo recurre a las interrelaciones en el lado de la producción.

Estrechamente ligado a este punto de vista hay un aspecto adicional: abordar la dicotomía clásica entre "valor de uso" y "valor de cambio" tal como la formularon los fundadores de la economía moderna, en particular Adam Smith y David Ricardo. Sraffa y Dobb tenían gran interés en este problema ${ }^{16}, \mathrm{y}$ ahora paso a ese tema.

\section{USO, INTERCAMBIO Y CONTRAFACTUALES}

E1 libro fundamental de David Ricardo, Principios de economía política y tributación, publicado en 1817, empieza con el siguiente pasaje:

Adam Smith observó que la palabra "valor" tiene dos significados diferentes, pues a veces expresa la utilidad de un objeto particular y otras, la capacidad de comprar otros bienes, la cual proviene de la posesión de dinero. Al primero lo podemos llamar "valor de uso" y al segundo "valor de cambio". "Las cosas", sigue diciendo, "que tienen gran valor de uso, comúnmente tienen poco o ningún valor de cambio, y, por el contrario, las que tienen gran valor de cambio, tienen poco o ningún valor de uso". El agua y el aire son sumamente útiles; son, además, indispensables para la vida; sin embargo, en circunstancias comunes, nada se puede obtener a cambio de ellos. E1 oro, en cambio, a pesar de tener poco uso, en comparación con el aire o el agua, se puede intercambiar por una gran cantidad de otros bienes.

Aquí hay un acertijo de interés en sí mismo y que nos puede decir algo sobre cómo podemos pensar en los precios y valores en general. Hay dos maneras alternativas de explicar de modo comprensible por qué el oro puede llegar a tener un precio mayor que el agua, a pesar de que es mucho menos importante para la vida humana. Una respuesta, basada en el lado de la utilidad de la foto, es que dadas la gran cantidad de agua disponible y la escasez de oro, la "utilidad marginal" del agua (el beneficio adicional que obtiene un consumidor de una unidad adicional de agua) es pequeña, en comparación con la utilidad marginal del oro. La otra respuesta es que el costo de producción del oro -o de extraerlo de la mina- es mucho mayor que el del agua, en esa situación dada de la economía.

Ninguna de ellas intenta explicar causalmente por qué y cómo surgieron los precios y las cantidades que se observan en la realidad. Son, más bien, respuestas a la pregunta de Smith-Ricardo: ¿por

${ }^{16}$ Ver Ricardo (1951-1973), editado por Sraffa con la ayuda de Dobb; y Dobb (1973). 
qué el oro, "aunque de poco uso en comparación con el aire o el agua”, se intercambia "por una gran cantidad de otros bienes"? La explicación basada en el costo y la explicación basada en la utilidad son, entonces, maneras alternativas de explicar lo que observamos, recurriendo a ideas como costos de producción y utilidad marginal que pueden servir como medio de comunicación social y comprensión pública.

Aunque Sraffa no publicó mucho que se relacione directamente con este problema de interpretación (salvo para comentar una distinción que implica el uso de conceptos "contrafactuales", que tratamos más adelante), podemos tener una idea de los problemas involucrados a partir de los escritos de Maurice Dobb, su amigo, colaborador y exponente. En un artículo clásico sobre "los requisitos de una teoría del valor", incluido en Economía política y capitalismo (1937), argumentó que la teoría del valor no se debe considerar tan solo como un artefacto mecánico que tiene un simple uso instrumental en la teoría de los precios. Aunque las teorías del valor abordan la "pregunta de Smith-Ricardo" respecto a una comprensión coherente de la estructura dual del valor de uso y el valor de cambio, intentan hacer afirmaciones sociales, importantes en sí mismas, sobre el carácter del mundo económico centrándose respectivamente en asuntos como la utilidad incremental de las mercancías, la satisfacción que pueden proporcionar, el trabajo usado en su fabricación o los costos en que se ha incurrido para producirlas.

Es bien conocida la inclinación de la economía política clásica, incluida la economía marxista clásica, a esperar de la teoría del valor algo más que una teoría de precios mecánica basada en los "productos intermedios". Esta inclinación a menudo se toma como una defensa especial, por razones políticas, de una justificación artificiosa de la relevancia de la teoría del valor trabajo. Pero esta interpretación no hace justicia a la visión clásica porque la importancia de la explicación comprensible y la comunicación forman parte del enfoque clásico. En este contexto es necesario recordar el significado que comúnmente se ha atribuido, en la visión de la economía política clásica y marxista, no solo al trabajo y la producción, sino también a la idea de "valor de uso" (y al concepto posterior de satisfacción -o "utilidad"- que las mercancías pueden proporcionar). La comparación entre las dos teorías rivales del valor - del valor trabajo y de la utilidad- fue considerada de interés justamente porque ambas hacían afirmaciones socialmente comprometidas; aquí no se intenta negar el interés social de la teoría de la utilidad como teoría del valor. 
Por cierto, en 1929, en una crítica profética de lo que después se convertiría en el enfoque de las "preferencias reveladas" (encabezado por Samuelson, 1938), Dobb lamentó la tendencia de la economía moderna a restar importancia a los aspectos psicológicos de la utilidad en favor de la simple elección:

La tendencia actual de la teoría moderna es abandonar [...] las concepciones psicológicas: a hacer que la utilidad y la desutilidad coincidan con la oferta observada en el mercado, a abandonar la "teoría del valor" en favor de la

“teoría del precio". Pero eso es capitular, no resolver el problema $(1929,32)^{17}$.

"El problema” al que se refiere Dobb, para el que son apropiadas las teorías de la utilidad y del valor trabajo, es hacer "una importante afirmación cualitativa sobre el carácter del problema económico" (Dobb 1937,21-22). Dobb pasó a diferenciar estas dos explicaciones sociales señalando que "la afirmación cualitativa [teoría de la utilidad] era de un orden muy diferente, no se refería a las relaciones de producción, sino a la relación de las mercancías con la psicología de los consumidores" (ibíd., 21). En cambio, la foto de la economía que presenta Sraffa se concentra precisamente en "las relaciones de producción", y al explicar los aportes de Sraffa, Dobb (1973) continúa este contraste.

Hay muchas pruebas de que este contraste era de gran interés para Sraffa. Pero en esta comparación, Sraffa vio otra gran diferencia que para él era metodológicamente importante (aunque no conozco pruebas de que le interesara mucho a Dobb), debido a su sospecha filosófica contra el uso de magnitudes "contrafactuales" en las descripciones factuales. Sraffa señaló que al optar por una explicación basada en el costo (acorde con Sraffa, 1960), podemos confiar totalmente en hechos "observados", como los insumos y los productos y una tasa de interés dados, sin tener que invocar "contrafactuales" (es decir, sin tener que suponer lo que habría ocurrido si las cosas hubiesen sido diferentes $)^{18}$. Este no es el caso en la explicación basada en la utilidad, pues la "utilidad marginal" implica inevitablemente un razonamiento contrafactual, porque refleja cuánta utilidad adicional se tendría si hubiese una unidad más de la mercancía.

El estatus filosófico de los contrafactuales ha sido objeto de mucho debate en epistemología. Veo poco mérito en el intento de excluirlos

\footnotetext{
${ }^{17}$ Dobb $(1929,32)$. También es de interés señalar que en una carta a R. P. Dutt, otro intelectual marxista, Dobb escribió el 20 de mayo de 1925 (poco después de su primer encuentro con Sraffa): "la teoría de la utilidad marginal me parece perfectamente sólida, y como explicación de los precios y los cambios de precio un avance frente a la doctrina clásica, dándole un marco más preciso y creando un instrumento de análisis más exacto”; ver Pollit (1990).

${ }^{18}$ Ver Sraffa $(1960, v-v i)$.
} 
al tratar de entender el mundo ${ }^{19}$. Pero sé -por largas conversaciones con Sraffa- que él consideraba que el uso de contrafactuales implicaba dificultades que las proposiciones basadas en la observación no ocasionaban. No es que él nunca usara conceptos contrafactuales (la vida habría sido insoportable con esa abstinencia) sino que pensaba que allí había una gran línea divisoria metodológica. Se esté o no de acuerdo con el juicio de Sraffa sobre la falta de fiabilidad de los contrafactuales, es notable que haya tal contraste metodológico entre las historias basadas en la utilidad y las basadas en el costo (en su forma sraffiana). La diferencia entre ellas no reside simplemente en el hecho de que la primera se centre en las condiciones mentales, en la forma de la utilidad, mientras que la segunda se concentre en las condiciones materiales de producción (un contraste que es fácil ver y que ha sido muy discutido); sino también en la distinción menos conocida de que la primera debe recurrir a contrafactuales mientras que la segunda -en la formulación sraffiana- no tiene esa necesidad.

\section{OBSERVACIONES FINALES}

El papel esencial de Piero Sraffa por su contribución a los profundos cambios de dirección en la filosofía contemporánea, ayudando a persuadir a Wittgenstein de que pasara del Tractatus a la teoría que después expuso en Investigaciones filosóficas, es muy reconocido por el mismo Wittgenstein (y por sus biógrafos). Lo que puede parecer intrigante, sin embargo, es que Sraffa no se emocionara por la trascendencia de esta influencia y la novedad de las ideas subyacentes. Sin embargo, la dificultad del acertijo se atenúa en gran medida cuando se sabe que esos temas eran parte de la discusión común en el círculo intelectual italiano al que perteneció Sraffa, y que incluía a Gramsci.

Por ello, la debilidad de la visión del significado y del lenguaje que Wittgenstein expuso en el Tractatus no sorprendió a Sraffa, y tampoco la necesidad de invocar consideraciones que luego se $\mathrm{co}^{-}$ nocieron como la "manera antropológica" de entender el significado y el uso del lenguaje. Parece haber una evidente "conexión Gramsci" en el paso del primer Wittgenstein al segundo Wittgenstein, aunque se necesitaría mucha más investigación para separar, si es posible, los aportes respectivos de Sraffa y de Gramsci a las ideas que surgieron en su círculo intelectual.

\footnotetext{
${ }_{19}$ Por cierto, el alcance de la economía como disciplina sería muy limitado si se prohibiese todo razonamiento contrafactual, como intenté mostrar en Sen (2002); ver también Sen (1982, ensayo 20, 432-449).
}

Revista de Economía Institucional, vol. i8, N. ${ }^{\circ} 34$, Primer Semestre/2oi6, Pp. 257-220 
Volviendo a los aportes económicos de Sraffa, en general no se pueden divorciar de su visión filosófica. Después de sus primeros escritos sobre teoría de la firma (y de su demostración de la necesidad de considerar la competencia "imperfecta" o "monopolista"), su obra posterior no se ocupó de buscar respuestas diferentes a las preguntas comunes de la economía tradicional, sino de alterar -y en cierto modo ampliar- el tipo de investigación al que se dedicaba la economía tradicional. En este ensayo he argumentado que el camino que siguió Sraffa se puede interpretar en términos de la función comunicativa de la teoría económica en asuntos de interés descriptivo general (y no como un intento de construir una teoría causal alternativa de la determinación de los precios y la distribución $)^{20}$.

Sraffa empleó el razonamiento analítico para arrojar luz sobre temas de discusión pública en contextos políticos y sociales. En particular, demostró que era inviable considerar que las ganancias son un reflejo de la productividad del capital. Más constructivamente, la obra de Sraffa arroja luz sobre la importancia de la teoría del valor en la descripción comprensible. El contraste entre interpretación de los precios basada en la utilidad y basada en los costos pertenece al mundo de la descripción y la discusión social pertinentes, y las descripciones rivales son de interés general; se invocaron en el pasado y hoy siguen siendo relevantes. La investigación de descripciones alternativas difiere de la determinación causal de los precios, en la que tienden a participar simultáneamente la demanda y la oferta.

Existe aquí una semejanza obvia con la aclaración clásica de John Hicks $(1940,1981)$ de que si bien la utilidad y los costos son necesarios en una teoría de la determinación del precio, cuando se trata de "valorar el ingreso social", la utilidad y los costos proporcionan dos maneras alternativas de interpretar los precios, con implicaciones diferentes en la comprensión del ingreso social o nacional. La medición del ingreso social "en términos reales puede significar valoración en términos de utilidad, o en términos del costo, y estos dos significados son en principio diferentes" (Hicks, 1981, 142) ${ }^{21}$.

\footnotetext{
${ }^{20}$ Puesto que el libro clásico de Sraffa tiene el subtítulo "Preludio a una crítica de la teoría económica", existe la tentación de suponer que una vez completara la "crítica" - de la cual el libro es un "preludio"-, Sraffa esperaba plantear una teoría alternativa de los precios y la distribución. Si los argumentos que se exponen en este ensayo son correctos, esa presunción es errónea. Según este punto de vista, Sraffa intentó ampliar el alcance y el ámbito de la investigación económica y no exactamente encontrar respuestas diferentes a las preguntas comunes de la teoría económica tradicional.

${ }^{21} \mathrm{E} 1$ amplio alcance del contraste hicksiano entre estas dos visiones alternativas es uno de los temas que se exploran en Sen (1979). En un comentario sobre su artículo de 1940, Hicks señala: "hoy pienso que [...] exigí muy poco a la medi-
} 
En la búsqueda de la distinción descriptiva entre utilidad y costos, Sraffa atribuyó importancia a la demostración de que su explicación de la historia basada en los costos se basa únicamente en información observada, sin tener que invocar supuestos contrafactuales. Esto difiere de la foto basada en la utilidad, porque el concepto de utilidad marginal es intrínsecamente contrafactual. Cuán importante metodológicamente es esta distinción -entre descripciones con o sin contrafactuales- es una pregunta abierta (confieso que sigo siendo escéptico), pero es un tema al que Sraffa atribuyó gran importancia. También se relaciona con otros rasgos de su análisis metodológico, incluida su extenuante pero correcta insistencia en que su análisis no necesita el supuesto de rendimientos constantes a escala ${ }^{22}$.

Se debe rechazar la tentación de ver la contribución de Sraffa como una teoría causal de la determinación del precio (arreglándoselas, misteriosamente, sin dar un papel a las condiciones de demanda). Aquí, todo gira sobre el significado de "determinación" y el uso que él da a ese término. El sentido de "determinación” que usa Sraffa se refiere a la determinación matemática de un conjunto de hechos a partir de otro conjunto. Para ilustrar el punto (con un ejemplo extremo), un reloj de sol puede permitirnos "determinar" qué hora es mirando la sombra del indicador (gnomon), pero no es que la sombra del indicador "determine causalmente" qué hora es. E1 valor de un reloj no reside en su capacidad para "fijar" la hora del día sino en "decirla".

Habría sido muy sorprendente que, en su análisis económico, Sraffa no estuviese influido por su propia posición filosófica, y que se hubiese mantenido dentro de las limitadas fronteras del razonamiento positivista o descriptivo comúnmente invocado en la economía hoy predominante. Al tratar temas económicos fundamentales de interés social y político general (algunos de los cuales se han discutido durante doscientos años), Sraffa fue mucho más allá de esas fronteras

da del costo" $(1981,143)$. Una exploración pionera de la valoración del ingreso nacional basada en la producción, y no en la utilidad, se encuentra en Mirrlees (1969). Como esa valoración implica investigar posibilidades de producción para hacer comparaciones del ingreso real (un ejercicio esencialmente contrafactual), el análisis de Mirrlees va en una dirección muy diferente de la investigación de Sraffa de las relaciones internas en el lado de los costos, para una situación de producción dada. Solo hay semejanza en (1) que no se necesita una teoría completa de la determinación causal de los precios para valorar el ingreso social o para usar la teoría del valor en una descripción social de la utilidad o los costos, y (2) que la separación de la historia de los costos de la historia de la utilidad está involucrada en ambos ejercicios.

${ }^{22}$ Ver Sraffa (1960, v-vi). Sraffa dice: "en 1928, cuando Lord Keynes leyó un borrador de este escrito, recomendó que si no se iban a suponer 'rendimientos constantes', se debía hacer una advertencia enfática a ese respecto (p. vi). Esa "advertencia enfática" se encuentra en el prólogo. 
estrechas. Supongo que es reconfortante saber que no había muchos Piero Sraffa sino uno solo.

\section{REFERENCIAS BIBLIOGRÁFICAS}

1. Albani, P. "Sraffa and Wittgenstein: Profile of an intellectual friendship”, History of Economic Ideas 6, 3, 1998, pp. 151-173.

2. Baranzini, M. y G. C. Harcourt, eds. The dynamics of the wealth of nations: Growth, distribution and structural change. Essays in honour of Luigi Pasinetti, Nueva York, St. Matin's Presss, 1993.

3. Bharadwaj, K. "Sraffa's Ricardo", Cambridge Journal of Economics, 12, 1, 1988, pp. 67-84.

4. Bharadwaj, K. "Sraffa's return to classical theory", K. Bharadwaj y B. Schefold, eds., Essays on Piero Sraffa: Critical perspectives on the revival of classical theory, Londres, Routledge, 1990, pp. 53-81.

5. Bharadwaj, K. y B. Schefold, eds. Essays on Piero Sraffa: Critical perspectives on the revival of classical theory, Londres, Routledge, 1990.

6. Blackburn, S. Dictionary of philosophy, Nueva York, Oxford University Press, 1994.

7. Bliss, C. J. Capital theory and the distribution of income, Amsterdam, North-Holland, 1975.

8. Burmeister, E. Capital theory and dynamics, Cambridge, Cambridge University Press, 1980.

9. Chamberlin, E. H. The theory of monopolistic competition, Cambridge, Mass., Harvard University Press, 1933.

10. Coates, J. The claims of common sense: Moore, Wittgenstein, Keynes and the social sciences, Cambridge, Cambridge University Press, 1996.

11. Cohen, A. y G. C. Harcourt. "Whatever happened to the Cambridge capital theory controversies", Journal of Economic Perspectives, 17, 1, 2002, pp. 199-214.

12. Cozzi, T. y R. Marchionatti, eds. Piero Sraffa's political economy: $A$ centenary estimate, Londres, Routledge, 2000.

13. Davis, J. B. "Sraffa, interdependence and demand: The Gramscian influence", Review of Political Economy, 5, 1, pp. 22-39, 1993.

14. Davis, J. B. "Garmsci, Sraffa, and Wittgenstein: Philosophical linkages", European Journal of History of Economic Thought 9, 3, 2002, pp. 384-401.

15. Dobb, M. H. "A Sceptical view of the theory of wages", Economic Journal 39, 156, 1929, pp. 506-519.

16. Dobb, M. H. Political economy and capitalism, Londres, Routledge, 1937.

17. Dobb, M. H. On economic theory and socialism: Collected papers, Londres, Routledge, 1955.

18. Dobb, M. H. Theories of value and distribution since Adam Smith: Ideology and economic theory, Cambridge, Cambridge University Press, 1973.

19. Eatwell, J.; M. Milgate y P. Newman, eds. The New Palgrave: $A$ dictionary of economics, Londres, Macmillan, 1987.

20. Eatwell, J.; M. Milgate y P. Newman, eds. Capital theory, Londres, Macmillan, 1990. 
21. Eatwell, J. y C. Panico. "Sraffa, Piero (1898-1983)", J. Eatwell, M. Milgate y P. Newman, eds., The New Palgrave: A dictionary of economics, Londres, Macmillan, 1987, pp. 445-452.

22. Garegnani, P. Il capitale nelle teorie della distribuzione, Milán, Giuffrè, 1960.

23. Garegnani, P. "Heterogeneous capital, the production function and the theory of distribution", Review of Economic Studies, 37, 3, 1970, pp. 407-436.

24. Garegnani, P. "Quantity of capital”, J. Eatwell, M. Milgate y P. Newman, eds., The New Palgrave: A dictionary of economics, Londres, Macmillan, 1987.

25. Garegnani, P. "Sraffa: The theoretical world of the 'old classical economists", European Journal of History of Economic Thought 5, 3, 1998, pp. 415-429.

26. Gramsci, A. The modern prince and other writings, Londres, Lawrence Wishart, 1957.

27. Gramsci, A. Selections from the prison notebooks of Antonio Gramsci, Q. Hoare y G. N. Smith, eds., Londres, Lawrence Wishart, 1971.

28. Gramsci, A. Letters from prison, L. Lawner, trad. y ed., Londres, Jonathan Cape, 1975.

29. Hahn, F. H. "The neo-Ricardians", Cambridge Journal of Economic 6, 4, 1982, pp. 353-374.

30. Harcourt, G. C. Some Cambridge controversies in the theory of capital, Cambridge, Cambridge University Press, 1972.

31. Hicks, J. "The valuation of social income", Economica 7, 1940, pp. 104-114.

32. Hicks, J. Wealth and welfare: Collected essays on economic theory, vol. 1, Oxford, Blackwell, 1981.

33. Kaldor, N. "Piero Sraffa", Cambridge Journal of Economic 8, 1, 1984, pp. $1-5$.

34. Kaldor, N. "Piero Sraffa (1898-1983)", Proceedings British Academy 71, 1985, pp. 615-640.

35. Keynes, J. M. y P. Sraffa. "Introduction", An abstract of a Treatise of human nature 1740. A pamphlet hitherto unknown, by David Hume, J. M. Keynes y P. Sraffa, eds., Cambridge, Cambridge University Press, 1938.

36. Kripke, S. A. Wittgenstein on rules and private language, Cambridge, Mass., Harvard University Press, 1982.

37. Kurz, H. Capital, distribution and effective demand: Studies in the classical approach to economic theory, Cambridge, Polity Press, 1990.

38. Kurz, H., ed. Critical essays on Piero Sraffa's legacy in economics, Cambridge, Cambridge University Press, 2000.

39. Kurz, H. y N. Salvadori. "Piero Sraffa's contributions to economics: A brief survey", H. Kurz, ed., Critical essays on Piero Sraffa's legacy in economics, Cambridge, Cambridge University Press, 2000, pp. 3-24.

40. McGuinness, B., ed. Wittgenstein and his times, Oxford, Blackwell, 1982.

41. Mirrlees, J. A. "The evaluation of national income and an imperfect economy", Pakistan Development Review, 9, 1, 1969, pp. 1-13. 
42. Monk, R. Ludwig Wittgenstein: The duty of genius, Londres, Vintage, 1991.

43. Naldi, N. "Piero Sraffa and Antonio Gramsci: The friendship between 1919 and 1927", European Journal of History of Economic Thought 7, 1, 2000, pp. 79-114.

44. Pasinetti, L. L. "Changes in the rate of profit and switching of techniques", Quarterly Journal of Economic 80, 4, 1966, pp. 503-517.

45. Pasinetti, L. L. "Switches of techniques and the 'rate of return' in capital theory", Economic Journal 79, 1969, pp. 508-525.

46. Pasinetti, L. L. Growth and income distribution, Cambridge, Cambridge University Press, 1974.

47. Pasinetti, L. L. Lectures on the theory of production, Londres, Macmillan, 1977.

48. Pasinetti, L. L. "Sraffa, Piero", International Encyclopedia of Social Sciences, vol. 18, Nueva York, Macmillan, 1979.

49. Pasinetti, L. L. "Sraffa on income distribution", Cambridge Journal of Economic 12, 1, 1988, pp. 135-138.

50. Pollit, B. H. "Clearing the path for 'Production of commodities by means of commodities': Notes on the collaboration of Maurice Dobb in Piero Sraffa's edition of the works and correspondence of David Ricardo", K. Bharadwaj y B. Schefold, eds., Essays on Piero Sraffa: Critical perspectives on the revival of classical theory, Londres, Routledge, 1990.

51. Potier, J.-P. Piero Sraffa - Unorthodox economist (1898-1983): A biographical essay, Londres y Nueva York, Routledge, 1987.

52. Ricardo, D. The works and correspondence of David Ricardo, 11 vols., P. Sraffa, ed., con M. H. Dobb, Cambridge, Cambridge University Press, 1951-1973.

53. Robinson, J. The economics of imperfect competition, Londres, Macmillan, 1933.

54. Robinson, J. "The production function and the theory of capital", Review of Economic Studies 21, 2, 1953-1954, pp. 81-106.

55. Robinson, J. "Prelude to a critique of economic theory", Oxford Economic Papers 13, 1, 1961, pp. 53-58.

56. Robinson, J. Economic philosophy, Harmondsworth, Penguin, 1964.

57. Roncaglia, A. Sraffa and the theory of prices [1978], 2. ${ }^{\mathrm{a}}$ ed., Nueva York, Wiley, 1981.

58. Roncaglia, A. Sraffa: la biografia, l'opera, le scuole, Roma y Bari, Laterza, 1999.

59. Salvadori, N. "Sraffa on demand: A textual analysis", H. Kurz, ed., Critical essays on Piero Sraffa's legacy in economics, Cambridge, Cambridge University Press, 2000, pp. 181-197.

60. Samuelson, P. A. "A note on the pure theory of consumers' behaviour", Economica 5, 1938, pp. 61-71.

61. Samuelson, P. A. "Parable and realism in capital theory: The surrogate production function", Review of Economic Studies, 29, 3, 1962, pp. 193-206.

62. Samuelson, P. A. “A summing up”, Quarterly Journal of Economics 80, 4, 1966, pp. 568-583. 
63. Samuelson, P. A. "Sraffian economics", J. Eatwell, M. Milgate y P. Newman, eds., The New Palgrave: A dictionary of economics, Londres, Macmillan, 1987.

64. Samuelson, P. A. "Revisionist findings on Sraffa", H. Kurz, ed., Critical essays on Piero Sraffa's legacy in economics, Cambridge, Cambridge University Press, 2000a, pp. 25-45.

65. Samuelson, P. A. "Sraffa's hits and Misses”, H. Kurz, ed., Critical essays on Piero Sraffa's legacy in economics, Cambridge, Cambridge University Press, 2000b, pp. 111-1152.

66. Schefold, B. Mr. Sraffa on joint production and other essays, Londres, Unwin Hyman, 1989.

67. Schefold, B. “Piero Sraffa 1898-1983”, Economic Journal 106, 438, 1996, pp. 1314-1125.

68. Sen, A. K. “On some debates in capital theory”, Economica 41, 1974, pp. 325-335.

69. Sen, A. K. "On the labour theory of value: Some methodological issues", Cambridge Journal of Economics 2, 1978, pp. 175-190.

70. Sen, A. K. "The welfare basis of real income comparisons: A survey", Journal of Economic Literature 17, 1, 1979, pp. 1-45.

71. Sen, A. K. Choice, welfare and measurement [1982], Oxford, Blackwell, reimpreso en Cambridge, Mass., Harvard University Press, 1997.

72. Sen, A. K. Resources, values and development, Cambridge, Mass., Harvard University Press, 1984.

73. Sen, A. K. Rationality and freedom, Cambridge, Harvard University Press, 2002.

74. Sen, A. K. "Piero Sraffa: A student's perspective", Atti dei convengni Lincei, vol. 200, Roma, 2004, pp. 23-60.

75. Smith, A. An inquiry into the nature and causes of the wealth of nations [1776], Oxford, Oxford University Press, 1976.

76. Solow, R. M. "The production function and the theory of capital", Review of Economic Studies 23, 2, 1955-1956, pp. 101-108.

77. Solow, R. M. Capital theory and the rate of return, Amsterdam, NorthHolland, 1963.

78. Sraffa, P. "Sulle relazioni fra costo e quantità prodotta", Annali di Economia 2, 1925, pp. 277-328, J. Eatwell y A. Roncaglia, trads., L. Pasinetti, ed., Italian Economic Papers, vol. 3, Bologna y Oxford, I1 Mulino y Oxford University Press, 1998.

79. Sraffa, P. “The laws of return under competitive conditions”, Economic Journal 36, 144, 1926, pp. 535-550.

80. Sraffa, P. 1951. "Introduction", The works and correspondence of David Ricardo (1951-1973), vol. I, pp. xiii-1xii.

81. Sraffa, P. Production of commodities by means of commodities: Prelude to a critique of economic theory, Cambridge, Cambridge University Press, 1960.

82. Steedman, I. Marx after Sraffa [1977], Londres, Verso, 1981.

83. Steedman, I. Sraffian economics, 2 vols., Aldershot, Elgar, 1988.

84. Sylos L., P. "Sraffa's critique of the Marshallian theory of prices", K. Bharadwaj y B. Schefold, eds., Essays on Piero Sraffa: Critical perspectives on the revival of classical theory, Londres, Routledge, 1990, pp. 3-19. 
85. Walsh, V. y H. Gram. Classical and neoclassical theories of general equilibrium, Nueva York, Oxford University Press, 1980.

86. Wittgenstein, L. Tractatus logico-philosophicus [1921], Nueva York, Routledge, 1961.

87. Wittgenstein, L. Philosophical investigations [1953], Oxford, Blackwell, 1958.

88. Wittgenstein, L. The blue and brown books, Oxford, Blackwell, 1958. 